of the orbit by it, but I think I could; in fact, I hardly ever call in the services of a general anaesthetist.

To our old-fashioned notions I know all this seems rather horrible, and many of you will think my plans of procedure and campaign somewhat extreme ; but, as John Hunter said, "don't think, but try."

Is one more liable to get intra-ocular choroidal haemorrhage, etc., by this method? Does the effort and exertion of walking home increase the risk? No, it does not; if anything it tends to reduce it by determining the blood to other parts and muscles called into action by the exercise. The cases of post-operation haemorrhage are due, of course, to a diseased condition of the blood-vessels unable to stand the rush of blood into them, and for the most part the bleeding comes on before the patient is off the table. Mr. Charles Higgens showed, at the Ophthalmological Society a good many years ago, three cases lost by haemorrhage following cataract extraction, and I had one in an only eye following iridectomy for acute glaucoma under a general anaesthetic years ago in a woman by no means a teetotaler.

Look at the advantages of the method: no assistant or nurse required; no preparations for general anaesthesia or vomiting and general disturbance following therefrom; no interference with the diet ; no struggling as the patient " goes under," no coming round of the patient and sudden movement when least expected; no anxiety due to sudden cessation of heart's action or respiration with artificial respiration and the mauvais quart d'heure following. The one little inconvenience is the prick of the needle during the injection; the site thereof is, of course, cocainised, but some slight pain is caused; occasionally a little faintness is produced, which is relieved by a dose of sal volatile or spirits.

\title{
RING INFILTRATION OF THE CORNEA IN A CASE OF PURPURA WITH A DISCUSSION OF ITS PATH- OGENESIS AND SOME GENERAL OBSERVATIONS ON THE DIFFERENT FORMS OF CORNEAL INFILTRATION*
}

BY

D. V. Giri, D.O. (Oxon),
Eastbourne.

Foreword

WHATEVER the incidence of ring infiltration or so-called ring abscess of the cornea after operations involving the introduction of

* Paper presented to the Oxford Ophthalmological Congress in July, 1917; written while the Author was Resident Surgeon to the Free Eye Hospital, Southampton. 
instruments into the eye and especially after flap-extraction of cataract, may have been in the pre-aseptic days, judging from the scant notice taken of it by the older authors, and the seriousness of the condition, unless one is inclined to be uncharitable to them, one is obliged to think that it must have been even then, not a particularly frequent complication of perforating wounds of the globe in the widest sense of the term. Since the inauguration of the era of asepsis and antisepsis by Lister, it has certainly become a very rare occurrence after operations. So far as I am aware, since 1875 - the year of publication of the first case of ring abscessliterature has record of only 20 cases, excluding the doubtful one of Hertel. Of these cases, 11 were of accidental wounds of the cornea caused by foreign bodies striking the eye-in one instance, recorded by Fuchs, the ring abscess occurring as a late infection taking its origin in an iris incarcerated in the sclera, as the result of an injury from a large needle three years previously; 4 occurred after cataract extraction, and the remaining 5 in the course of metastatic ophthalmia. None of the 11 cases of Fuchs, or the 4 cases of Collins were of metastatic origin. Every one of the 5 metastatic cases had a localized focus of sepsis in some part of the body or other. Weiss's case was in a patient suffering from complicated fracture of the femur. Hirschberg's patient was a man ailing from suppurative cystitis in addition to tabes dorsalis, etc. Herrenheiser's case and Wintersteiner's referred to by Fuchs, were in women suffering from puerperal septicaemia. Axenfeld's case was one of cryptogenetic pyaemia after cholelithiasis without liver abscess ; the patient, a woman, presented endocarditis ulcerosa, meningitis suppurativa metastatica, and multiple abscesses in the substance of the brain as metastatic foci in addition to the eye. Of the 20 cases, 12 were in men and 8 in women. Out of the 12 cases in men, as many as 10 were due to accidental wounds of the cornea, and 2 of metastatic origin. Only 1 out of the 8 cases in women was due to accidental trauma, 4 resulted from wound infection after cataract extraction, and 3 accounted for by metastasis. This is just as one would expect in view of the greater exposure of men to the risk of foreign bodies, especially metallic ones, hitting the eye in the course of their occupation in steel, iron, and other metal works, the rarity of wound infection after operation-because of asepsis and antisepsis-and the peculiar liability of women to puerperal infection. The ages of the patients ranged from 23 to 70 , the great majority being between 30 and 50 .

From the above analysis it is evident that hitherto there has been no patient well within the teens in whom ring infiltration of the cornea has been reported, and no case of an acute general infection giving rise to the condition. Hence arises the special interest 
of the following case which presents other noteworthy points perhaps best considered after the description of the facts.

\section{Case}

James Cole, 13 years of age, was admitted into the Free Eye Hospital, Southampton, on July 8,1916 . The history the mother of the patient gave was that a week previously, he returned from the swimming bath with a bad headache and was in bed for three days, during the first two of which he kept retching and had diarrhoea. He got up on the fourth day feeling better. He felt ill again on the fifth day and took to bed. The right eye got bad on the sixth day; , the trouble started with severe pain in the eye. She bathed the eye with milk and water to start with, and later with boric lotion. Spots on the body started coming on the fourth and following days. He had measles and broncho-pneumonia as a child. $\mathrm{He}$ is said to have attended the swimming bath every day for three or four days preceding his illness, and the mother surmises that it may have been due to a chill he may have caught in the bath.

State on admission.-I found the patient pale and very much run down. Patches of purpura all over the body; warts on the left hand which he has had for a long time. L. E. normal.

R. E., strong diffuse injection of the globe. Surface of the cornea stippled. Pupil small and inactive. Iris congested. Exudate in pupillary area. There is a yellowish-grey ring of infiltration of the cornea about $2 \mathrm{~mm}$. from the limbus. The central disc enclosed by the ring stands out, while the area between limbus and infiltration appears guttered. No red reflex.' P.L. and projection present, T.n. Teeth in good condition. Patient said not to be subject to constipation. Locally, a 1 per cent. ointment of quinin and atropin and hot boric fomentations were prescribed. My notes during his stay in the hospital are as follows:

July 9.- The infiltration extends all over the cornea, though densest in the ring. Has had hardly any pain in the eye. Left elbow swollen and movement limited. No sponginess of gums and no bleeding from them. Good appetite. Slept well. Heart normal. Local application of tincture of iodine and hot boric fomentations for the elbow.

July 10.-The cornea presents a smoother surface and is less dull. Urine : acid, concentrated; sp. gr. 1014 ; sugar and albumin, nil. July 11.-Bowels constipated. Mist. aperiens, З̌iss.

July 13.--Ring infiltration resolving. The ring much narrower and rarified. Rest of cornea clearer. Yellowish exudate projecting from the pupil. The cornea looks smaller. Though tension is not palpably lowered, the eye has a shrinking look.

July 14.-Tongue foul. Calomel gr. $\frac{1}{4}$ t.i.d. 
July 15.-Ring infiltration further absorbed. But for a dot in the centre the exudate in pupil also absorbed. T.n.

July 16.- Just a trace of the ring obvious. No red reflex. Faint P.L. No projection. T.n. Patches of purpura desquamating.

July 21. - No trace of ring. The lens itself appears clear. Whatever prevents the red reflex is behind the lens. Just a faint haze of the cornea. P.L. still present. T. - (?) Urine: faintly acid; sp. gr. 1010 ; sugar and albumin, nil.

July 27.-Cornea clear. A light yellow reflex from the back of the lens. P.L. only at about $18 \mathrm{~cm}$. Further away, light not perceived. No projection.

July 28.- Upper lid somewhat swollen. Eye tender. Superficial vessels growing over cornea at outer and lower quadrant. Discharged. Hydrarg. $\overline{\mathrm{c}}$ creta, gr. ii, t.i.d internally. Ung. atrop. $1 \%$ t.i.d. locally. A temperature chart was maintained from July 10 to July 25. He ran a febrile temperature from July 10 to July 20, with a range of from $1^{\circ}$ to $2^{\circ} \mathrm{F}$.

August 8.- Seen in the out-patient department. Iris pushed forward. Yellow reflex from the back of the lens more striking. No P.L. Eye again strongly injected and watering.

August 11.-Because of its having become a soft blind eye which would not quiet down, the patient was re-admitted and the eye excised under general anaesthesia.

Macroscopic pathological examination.-The eye was cut open antero-posteriorly from the corneal limbus to the stump of the optic nerve. The eye contained clear, watery fluid. The retina was completely detached and shrivelled up behind the disc of plastic exudate overlying the back of the lens and the ciliary body in its entirety. The detachment was evidently due to the shrinking exudate pulling on the retina. The advancing of the iris and the extreme shallowing of the anterior chamber is accounted for partly by its being pushed forward along with the lens by the contracting plastic exudate behind, and partly by the cessation of formation of the aqueous, owing to the disorganization of the ciliary body and processes during the course of the inflammation.

\section{Remarks}

In this instance the purpura was characterised by lesions in the skin, swelling of one elbow with limitation of movement, and gastrointestinal disturbances. This is a form of the disease affecting children, and known as "Henoch's purpura." Whether or not this variety of the malady is particularly prone to involve the eye cannot obviously be decided on the strength of a single case. Seeing that there was no history of scarlet fever or rheumatic fever, that the heart and kidneys were normal, the teeth and gums were healthy, and there was no localized focus of sepsis discoverable elsewhere, it 
is highly probable, if not certain, that the affection of the eye was a local manifestation of the general disease of the blood, and not a mere coincidence.

Here, as noted by previous observers, the annular infiltration was characterized by the rapidity of its formation, a complete, $\downarrow$ wellformed ring having occurred within 48 hours after the first manifestation of ocular symptoms. Unlike what is usual after ring infiltration of the cornea, in this instance the infiltration retrogressed completely. The eye did not go on to panophthalmitis with concomitant increase of tension, but became soft and shrunken owing to the cessation of formation of the aqueous, detachment of the retina, and disorganization of the vitreous and ciliary body in the course of the plastic iridocyclitis.

It may not be out of place here to discuss the pathogenesis of ring infiltration of the cornea in particular and incidentally of other forms of corneal infiltration in general. The first anatomical description of the condition was given by Fuchs in 1881 as the result of examination of the cornea of a diabetic woman aged 30 years. On the third day after linear extraction of cataract the ring abscess began to form, and four days later the whole cornea got sequestrated as a necrosed disc; it permitted of being lifted off the sclera as if it had been divided with a knife along its border. The next anatomical description of the condition was by Collins in 1893 . Collins says that he was first led to an examination of the cases and specimens, of which he gives the details, by the interesting theory of the pathogenesis of this condition given by Leber, and that he was exceedingly sorry to find that instead of supporting it, they offer serious objections to its acceptance. To quote Collins's summary of Leber's explanation of ring infiltration of the cornea : "He considers that when microbes are inoculated into the cornea, their action is not limited to the small area which they occupy, but that a kind of distant action takes place, due to the diffusion of irritating substances. In this way, what he terms a microbic area is formed around the seat of the wound. White blood corpuscles migrate from the vessels at the corneal margin and make their way towards the microbic area, without, however, quite reaching it, as it offers a resistance to their further advance. Thus a ring of infiltration is formed, the changes in the microbic area itself being simply necrotic in character. It follows that if this theory be true, we ought always to find the wound situated nearly in the centre of the ring of infiltration ..." As in cases of ring infiltration of the cornea after perforating wounds, the stuation of the annulus is invariable, whereas the situation of the wound is, oftener than not, anywhere but the centre of the ring, Collins thinks that Leber's theory is in conflict with the reality. He does not attempt to offer an explanation of the phenomenon himself. Fuchs refers 
in his work to Collins's cases and contention, and in elucidation of the peculiar distribution of the infiltrating cells, says: "The ring abscess was designated as the immigration ring (Einwanderungsring).* In experiments on the cornea of the rabbit it lies in the form of a circle around the inoculated spot. In contradiction to it, the ring abscess in man by no means corresponds in its position with the position of the wound, which may even be outside the ring of pus. This is because it is not a ring of immigration to the corneal wound but to the necrotic cornea, and has therefore the same position wherever the wound may be situated ..." Parsons shares Fuchs's view. Here it would be well to look into what Leber says : "Allow me for further explanation to describe to you a keratitis caused by inoculation of micro-organisms. Suppose the microbe has developed in the centre of the cornea, so as to occupy a certain area still remote from the corneal margin. The effect in the area directly attacked will consist only of regressive changes in the cells resulting in necrosis; necrosis and exfoliation of the anterior epithelium; loss of the endothelium covering the posterior surface; and decay of the stellate cells of the cornea which is recognized by loss of their processes, vesicular degeneration of the protoplasm, and loss of the staining property of the nucleus.

But at first, if the action of the microbe is sufficiently intense, neither migration of the leucocytes nor proliferation of the cornea cells takes place

Whilst thus within the microbic area itself, and its immediate neighbourhood, only necrotic changes are to be seen, at some distance from it there appears a zone of dense infiltration of pus corpuscles, which aftects more or less the entire thickness of the cornea. . . The pus corpuscles which form the ring-like infiltration of the cornea are, according to the investigations of Waller and Cohnheim, white blood corpuscles which have migrated from the vessels at the corneal margin, and have made their way towards the microbic area, without, however, quite reaching it. . ."

Another experiment is founded upon the already mentioned observation, that the purulent infiltration of the cornea takes place at a distance round the microbic area. Thus pus cells migrating from the corneal margin meet with a resistance on the part of the microbic area to their further advance.

Those who support the view that the pus cells are produced by proliferation of the corneal corpuscles, must explain this appearance by supposing that the corneal corpuscles in the neighbourhood of the microbic area perish, while at some distance from the same they are stimulated to proliferation. Which of the two views is correct can easily be shown by inoculating the microbes in two or more

N.B.-In his Bowman Lecture, Leber does not mention what animal he experimented in this connection. 
separate places between the centre and margin of the cornea where the action of the different colonies would be accumulated. But if the purulent infiltration of the cornea depends upon the marginal emigration, then the infiltration will commence first in the neighbourhood of the corneal margin, and the separate zones of infiltration perhaps never form, because the pus cells find a great resistance to their advancement in the spaces between the single colonies.

The facts completely confirm the latter theory; for separate infiltration rings are not found, but only one common zone of infiltration near the corneal margin, surrounding all the separate colonies.

It follows from these deductions that the action of the microbes, as I have mentioned before, is not limited to the small area which they occupy, but that a kind of distant action takes place which finds its simple explanation in the diffusion of irritating substances." That irritating substances act upon the tissues of the body by diffusing through them is proved by Leber experimentally.

In view of what I have quoted above from Leber and the criticisms of his deductions preceding it, I do not feel convinced that he has been properly understood. What Collins takes Leber to mean does not appear to me to follow from what Leber says. As we have already seen, in refuting the view held by some that the pus cells originate in the corneal cells, Leber makes it clear that in every case the wound or the focus of infection does not necessarily form the centre of the circle of infiltration in the mathematical sense. Fuchs himself mentions that in some cases the formation of the ring of infiltration takes more time and that the process terminates, not in the necrosis, but in permanent opacity and flattening of the cornea. In one of his cases in which 48 hours after a perforating injury of the cornea ring infiltration was noted, the wound was thoroughly cauterized with the galvano-cautery, and the grey ring retrogressed gradually, leaving at the end of a fortnight a clear cornea but for the scar at the site of the wound. Similarly, in the instance I have described above, the infiltration disappeared completely also in the course of about a fortnight. In view of these facts, the explanation that the annulus of infiltration is not a ring of immigration to the corneal wound but to the necrotic cornea, is not quite obvious; surely, if the necrosis of the tissue were the cause of the infiltration the cases in which the whole cornea survives will have to be looked upon as instances of resuscitation of dead tissue following on the infiltration called forth by it-a by no means tenable view, which I do not think Fuchs intended to convey: For even at the time of advancing this explanation of the leucocytic immigration ring, Fuchs himself does not appear to have been altogether satisfied with it. In the latter part of the same communication- " On Ring Abscess of the Cornea"-he says, "It is conceivable that the 
injurious substances which penetrate the cornea from the anterior chamber do not really harm it sufficiently to make it necrotic, but are quite sufficient to call forth the ring of immigration. In this manner are to be explained the cases with a mild course, which go on, not to suppuration, but merely to opacification and flattening of the cornea, or even to complete clearing of the cornea, as in the case of healed ring abscess mentioned at the outset. . . ." A' little further on, after citing a case of melano-sarcoma of the choroid attended with high increase of intra-ocular pressure, in which pathological examination of sections of the excised eye showed absence of endothelium in the pupillary area, and a ring of infiltration in the cornea, he says: "In this instance, as in the case of the genuine ring abscess, the ring of immigration is to be attributed, not to a circumscribed lesion, as in experiments on the rabbit, but to the injury of the cornea as a whole." This statement contains an inkling of what appears to me to be the explanation of ring infiltration. Thanks to the labours of Wright, Metchnikoff, and others in the field of immunity, since the time of Leber's work on inflammation and Fuchs's publication on ring abscess, our knowledge of the processes of inflammation has been made clearer. In the light of this new knowledge, which I find already foreshadowed in the wonderfully comprehensive work of Leber when he says . . . "But the proof is not yet furnished that the microbes are harmless because they have been taken up by the cells, and that they may not, on the contrary, have been taken up because they were already killed or debilitated by other influences . . . " More recent investigations have further shown that the power of the body to kill the microorganisms or to render them harmless, is not alone the property of the leucocytes, for it appears that the blood and other tissues or fluids possess this power in a still higher degree," and the data furnished by Fuchs's work, one is enabled to offer what appears to my mind a not unacceptable explanation of the phenomenon of ring infiltration. Essentially it is a ring of infiltration, neither to the wound nor to the necrotic cornea, but to the toxin-pervaded area of the cornea, which invariably involves the whole of this transparent structure. In the first instance the leucocytes are called forth to save the attacked tissue. But as sometimes a physician, called in at the eleventh hour to save an individual, may find on arrival that he is either unable to tide the patient over the crisis, or that the only thing left for him to do is to issue the death certificate, so it devolves often on the leucocytes in ring infiltration to deal with dead tissue. Let us first consider cases of the condition resulting from perforating wounds of the cornea. Why does the annulus of infiltration invariably occupy the same position, irrespective of the situation of the wound ? Because of the cornea being an island of avascular tissue, it is inevitable. We 
know that no entry of leucocytes from the anterior chamber into the cornea can take place so long as Descemet's membrane is intact. Fuchs has pointed out that he has never seen in ring-abscess the splitting into layers and destruction of Descemet's membrane, as described by Elschnig in the case of posterior abscess. Hence, we must look wholly to the limbal loops and anterior ciliary vessels for the source of the infiltrating leucocytes. In order to excite emigration of leucocytes, it is necessary that the vessel should be acted upon by the phlogogenetic agent, producing what, according to Leber, may be looked upon as a toxic paralysis of its cells and loss of tone of its wall, with consequent dilatation. Suppose the wound is situated at the periphery of the cornea. Where the wound itself forms a focus of infection-it is hardly conceivable that it does not at the outset, although at a later date sections of the excised eye may not show any germs in it-the first vessels to come under the influence of the toxin produced at the focus of infection are those in the immediate neighbourhood of the wound, and the initial infiltration occurs in the area between the wound and the nearest point of the limbus to it. The movements of the leucocytes are determined by the structure of the tissue through which they have to pass, and by chemotaxis, which directs them towards the point of greatest concentration of the toxin. Because of these determining factors, the leucocytes do not carry out any encircling manœuvre in regard to the toxin-pervaded area, but are obliged to take the shortest possible route from the point at which they leave the vessel wall to the focus of greatest concentration of the toxin. Before emigration from the rest of the circumcorneal vessels can take place, the toxin has to diffuse through the whole of the cornea and act upon them, producing the necessary changes as it comes into contact with them one after another. How soon the whole cornea becomes permeated by the toxin depends upon the rapidity of output of the toxin by the invading bacteria and its power of diffusion. The process is accelerated by the toxin entering the anterior chamber from the wound, where the wound itself forms a bacterial base, and by the toxin formed in the anterior chamber itself by the germs carried into it and multiplied there acting through the aqueous simultaneously on the whole cornea from behind. The extent to which the leucocytes can progress towards the point of greatest concentration of the toxin depends upon its virulence. As it is only in cases of marked virulence of the infection, or low vitality of the organism, or both, that the phenomenon of ring infiltration is observed, and as the infiltration is not called forth from all sides until the whole cornea is already permeated in sufficient intensity by the toxin, it follows that the leucocytes meet with strong opposition to their progress at the very outset, and are 
early brought to a stop by the greater concentration of the toxin they encounter in going from the periphery towards the centre, where it cannot get diluted so easily as in the neighbourhood of the blood-vessels. When the front rank of leucocytes is arrested in its march, the succeeding ranks are obliged to mass behind it, so that a dense ring of leucocytes, instead of a diffuse infiltration, occurs. If the organism forms anti-toxins and bactericides sufficiently rapidly and the opsonic index is high, the toxins get more or less neutralized, the march of the leucocytes which had temporarily ceased is resumed, phagocytosis of the opsonised or killed bacteria occurs. The inflammatory process is stopped and the infiltration gets resorbed. Otherwise destruction of the invaded tissue occurs. In terms of warfare, the bacteria answer to the invading army, the toxins to the artillery of the invaders, the opsonins, anti-toxins, and bactericides to the artillery of the defenders, the action of the artillery preparing the way for the advance of the army-bacteria or leucocytes as the case may be-whose artillery proves superior in the combat.

Where ring infiltration of the cornea is of metastatic origin, or occurs in the course of a general acute disease of the blood, as in the case above, by the time the eye is aftected, the protective mechanism of the body, where its power of resistance is sufficiently high, is already more or less vigorously active, so that, in the case of the cornea, the attack and defence may be said to be simultaneous; only because of the attack taking place on the whole cornea at the same time through the aqueous, the toxin gets concentrated in the cornea, and the attack well established by the time the leucocytes emigrating from the circumcorneal vessels have advanced a millimetre or two from the limbus, and hence the check to their further progress and the formation of the annulus of infiltration.

Now let us turn to the consideration of other forms of corneal infiltration. In the concluding part of his communication on ring abscess of the cornea Fuchs draws a detailed contrast between serpiginous ulcer and ring infiltration as follows: "It is instructive to compare with each other the two fulminant bacterial affections of the cornea, ulcus serpens and ring abscess. As regards the clinical aspect, the following difterences arise: Ulcus serpens originates in superficial injuries of the cornea; the ring abscess occurs after perforating injuries, or without any injury whatever of the cornea. Ulcus serpens develops round the injured spot as its centre; the position of the ring abscess is always the same, irrespective of the situation of the injury. Ulcus serpens spreads from the centre towards the periphery, the ring abscess from the periphery towards the centre. Ulcus serpens spreads slowly and irregularly, the ring abscess very rapidly and uniformly. Ulcus serpens leads only very exceptionally, the ring abscess almost invariably, to panophthalmitis. Anatomically, the following 
differences present themselves: Ulcus serpens consists in the infiltration of the margins of the small loss of substance, while the ring abscess has nothing to do with the infiltration of the margins of the wound; in ring abscess it may either be present or absent. The ring abscess is a genuine ring of immigration, which cannot be said of ulcus serpens. In cross sections of the cornea the infiltration of the margins in ulcus serpens is wedgeshaped with the apex turned towards the periphery of the cornea. The reverse is the case in ring abscess, the cross section of the immigration ring being wedge-shaped with the apex directed towards the centre of the cornea. This corresponds with the opposed direction of propagation in the two forms of infiltration. In ulcus serpens the wedge-shaped infiltration of the margins is the main bacterial base, in ring abscess the annular infiltration never contains bacteria. In both diseases necrosis of the deep lamellae of the cornea takes place. In ulcus serpens the necrosis proceeds from the anterior side, in ring abscess from the posterior side of the cornea. In ulcus serpens immigration of pus from the exudate in the anterior chamber into the hindmost layers of the cornea frequently occurs, which is not the case in ring abscess. In ulcus serpens the exudate in the anterior chamber is germ free, in ring abscess rich in bacteria. That is why in this case the suppuration spreads into the interior and leads to panophthalmitis which occurs only exceptionally in ulcus serpens, if after extensive perforation the pyogenic organisms reach the interior. The usual causal agent of ulcus serpens is the pneumococcus. It is specially this organism that is, as a rule, not found in the interior of the eye in ring abscess but various other bacteria. It appears that ring abscess, just like panophthalmitis, could be produced by different species of bacteria."

This is in essence a contrast between a disease arising from a superficial injury and one arising from a deep injury or no injury at all; between superficial spurious infiltration and deep genuine infiltration. In the one case the infiltrating cells come from the conjunctival sac, until, at any rate, a late period in the disease, and in the other case from the blood-vessels of the circumcorneal zone from the outset. Why should the infiltration in the two cases differ ? In the first place the bacterial implantation in ring abscess is deep and extensive; in ulcus serpens it is superficial and minute. The course of ulcus serpens enables us to draw instructive inferences as regards the nature of the usual causal agent, the pneumococcus and its toxin. The organism does not multiply rapidly in the cornea, is slow in producing toxin. The toxin itself is incapable of quick diffusion, and of acting on the tissue of the cornea except in very high concentration. The situation of the ulcer being generally about the centre of the cornea, the toxin has 
to travel about 5 to $6 \mathrm{~mm}$. from where it is produced to the limbus, whereas it has only to diffuse through a thickness of about $1 \mathrm{~mm}$. to reach the anterior chamber. That is why hypopyon comes early into evidence in this disease, whereas deep infiltration which depends upon the circumcorneal vessels being affected by the toxin, comes late in its course, or may not occur at all, if in the meanwhile the ulcer retrogresses. Why the ulcer in most cases starts about the centre of the cornea is probably not because superficial injuries of the cornea do not occur in parts other than the centre, but because when situated near the limbus rich in blood-vessels, the microbe is too rapidly overwhelmed to produce mischief. These inferences as regards the nature of the infiltration, the position of the ulcer, and the nature of the pneumococcus and its toxin, are supported by the fact that not infrequently one sees individuals who have been going about for years with a chronically inflamed lacrymal sac continually discharging pneumococci into the conjunctival sac, without suffering from ulcus serpens. I venture to think it is common experience to ophthalmic surgeons in industrial districts to see patients who have had a foreign body impacted in the cornea sometimes for a week or more, with infiltration around the foreign body, developing no serpiginous ulcer. Probably more cases of foreign body in the cornea are dealt with in the workshop or factory itself, where the eye is not bandaged after the removal of the foreign body and no antiseptic ointment is put in, and the workman goes back to his work immediately, than are seen in eye hospitals, and yet how few are the cases of ulcus serpens arising from them?

The next important form of infiltration that remains to be dealt with is what one sees in interstitial keratitis. In this disease, why should the deep infiltration be diffuse and slow and not annular and rapid as in ring abscess? As has been already pointed out the movements of the infiltrating leucocytes are determined by the nature of the toxin that confronts them. Although the spirochaete of syphilis, which is mainly responsible for the disease, is very resistant to the defensive mechanism of the body, just like the other very occasional causal agent, the tubercle bacillus, and hence both very persistent in their action, their toxins are, as a rule, of low virulence, so that the leucocytes find no insurmountable obstacle to their advance. This explains why the infiltration is slow and diffuse, but for points of greater density of leucocytes here and there.

Keratitis disciformis is described by Fuchs as follows: "A keratitis, which is akin to ulcus serpens, is characterized by the development of a disc-shaped grey cloudiness in the middle layers of the cornea. As a rule, in the middle of the disc one sees a more strongly clouded fleck. The periphery of the disc is sharply defined by a deeper grey border. This disc-shaped infiltrate never turns yellow 
and never leads to destruction of the cornea. Only exceptionally a small loss of substance over a circumscribed area occurs. The signs of irritation are mostly not very marked; hypopyon is absent or very small. The course of the disease is prolonged, inasmuch as it takes a month or more before the eye becomes quite pale, and the infiltrate turns into a permanent opacity. . . Just as in ulcus serpens, the cause of the disease is an infection of the cornea from outside, the central deeper grey fleck representing the portal of entry of the bacteria. The epithelial lesion preceding the infection can be traced sometimes to a trivial injury and sometimes to herpes corneae febrilis, but in very many cases the cause remains unknown. . . . In keratitis disciformis the cloudiness appears uniformly grey, and only under strong magnification does it resolve itself into extremely fine, sharp, white densely placed dots. Further, the opacity is altogether sharply demarcated from the healthy cornea by the grey circular line at its border. . .." So far as I am aware, hitherto no investigation of the pathological anatomy of this condition has been published, which is only to be expected from the fact that it never calls for excision of the cornea or eye. To my mind it appears probable that the disc of cloudiness is not due to a leucocytic infiltration at all. The microbe, being of low virulence, dies off not long after its implantation into the cornea, producing during its short life a very small quantity of toxin, so small that it gets used up in diffusing to the extent of the well-defined grey disc. Such inflammatory reaction as occurs is obviously not strong enough to call forth leucocytic emigration from the circumcorneal vessels, as evidenced by the healthy cornea between the grey disc and the limbus. 'In all likelihood, what occurs is either opacification of the corneal fibres that the toxin comes into direct contact with, or the precipitation of opaque insoluble substances in the interstices, from the transparent lymph permeating the corneal tissue, by chemical action. This view is in some measure supported by the fact that in "Nodular opacity of the cornea," a non-inflammatory, dystrophic condition, first described by Groenouw, Fuchs has found by anatomical investigation that the opacity is due to precipitation of insoluble acidophile and basophile substances in the cornea.

My thanks are due to Mr. Zorab, surgeon to the hospital, for permission to publish the case.

References, in the order in which they occur, with pages quoted from.

Weiss, L.-Klin. Monatshl. f. Augenheilk., 1875, p. 393.

Hirschberg, J.-Cent.f. prak. Augenheilk., 1880, p. 188.

Herrenheiser, J.-Zeitschr. f. Augenheilk., 1893, Vol. XIV, p. I80.

Axenfeld, T.-Arch. f. Ophthal., Vol. XL, No. 3, p. 89.

5. Hare, H. B. - " A Text-Baok of the Practice of Medicine," 1905. London : Henry Kimpton. Purpura, p. 791. 
6. Collins, E. T.-Observations on Ring Infiltration of the Cornea. Ophthal. Review, Vol. XII, 1893, p. 221.

7. Fuchs, E.-Ueber Ringabscess der Hornhaut. Arch. f. Ophthal., 1903, V.ol. LVI, pp. I 3 (case vi.), 27, 28, 29.

8. Parsons, J. H.-Pathology of the Eye : Peripheral Annular Infiltration of the Cornea, p. 217.

9. Leber, T.-Bowman Lecture-On the Present l'osition of our Knowledge of Inflammation. Trans. Ophthal. Soc. U.K., Vol. XII, 1892, pp. I1, 12, I3, I4, 22,

Io. Muir, R. and Ritchie, G. - "Manual of Bacteriology," 1913. Immunity, p. 549.

I I. Fuchs, E._-"Lehrbuch der Augenheilkunde." Twelfth edition, I9Io. Keratitis disciformis, p. 270.

12. Fuchs, E. - Ueber Knötchenförmige Hornhauttrübung. Arch. f. Oththal., Vol. LXXXIX, second number, February, 1915, p. 337 .

\title{
HERPES ZOSTER AFFECTING THE CILIARY NERVES*
}

\author{
BY \\ GeORge W. Thompson, F.R.C.S. \\ LONDON
}

HERPETIC eruptions on the sclera may occur in the course of severe attacks of herpes zoster ophthalmicus affecting the branches of the first division of the fifth nerve, including its nasal branch, and are usually associated with such changes as herpetic vesicles of the cornea, keratitis striata, "keratitis punctata," and iritis. The efflorescences take the form either of small, reddish nodules, or of larger nodules, the centre of which is transparent, or even resembles a limpid vesicle. They usually appear about a fortnight after the corneal disturbances have declared themselves, and persist for one or two months longer than the latter. They eventually clear up, leaving a slight discolouration of the sclera, and an adhesion of the overlying conjunctiva. It is important that the underlying cause, be it syphilis, influenza, or nasal or dental trouble, be treated. When the acute manifestations have subsided under fomentations and atropin, yellow ointment with dionin should be employed, with a view to clearing up the scleral nodules.

The following four cases have been met with :-

Case No. 1.-A male patient, aged 41, suffered from left herpes zoster ophthalmicus, affecting most of the branches of the first division of the fifth nerve, on the day after his nose had been forcibly washed out by a throat specialist. When seen by me, ten days later, there were signs of herpes and anaesthesia on the left side of the nose, two small erosions on the upper part of the cornea, and typical "keratitis punctata"; and three days later keratitis striata was observed for the first time. Twenty-one days after the

*Abstract of a communication read at the 1918 Congress of the Ophthalmological Society of the United Kingdom. 